\title{
Distributed Space-Frequency Coding over Relay Channels
}

\author{
Karim G. Seddik and K. J. Ray Liu \\ Department of Electrical and Computer Engineering, \\ and Institute for Systems Research \\ University of Maryland, College Park, MD 20742, USA. \\ \{kseddik, kjrliu\}@umd.edu \\ July 15,2007
}

\begin{abstract}
In this paper, the design of distributed spacefrequency codes (DSFCs) implementing the decode-and-forward (DAF) protocol for wireless relay channels is considered. The proposed DSFCs are designed to achieve the frequency and cooperative diversities of the wireless relay channels. A two-hop system model, where there is no direct link from the source node to the destination node, is considered. We propose to use two stages of coding, namely, source node coding and relay nodes coding. The proposed DSFCs are proved to achieve full diversity of order $N L$ where $N$ is the number of relay nodes and $L$ is the number of paths per channel.
\end{abstract}

\section{INTRODUCTION}

Wireless channels have a lot of impairments such as fading, shadowing, and multiuser interference which can highly degrade the system performance. This has increased the thrill towards the study of wireless channels to overcome their impairments. Spatial diversity has proved to be an eminent candidate for combating the wireless channels impairments and hence, achieving the signal quality and high data rate promised by the future multimedia services. In wireless applications, it is affordable to have multiple antennas at the base station but it is difficult to equip the small mobile units with more than one antenna due to space constraints of the small mobile units. Hence, the use of multiple antennas at the mobile units is limited. This gave rise to what is known as cooperative diversity, in which the nodes try to form a virtual multiple element transmit antenna.

The techniques of cooperative diversity have been introduced, for example, by Sendonaris in the context of CDMA systems [1]. In [2], different protocols were proposed to achieve spatial diversity through node cooperation. Among those protocols are the decode-and-forward (DAF) and amplify-and-forward (AAF) protocols. In the decode-andforward protocol with one relay node, the relay node decodes the source symbol before re-transmitting to the destination. In the amplify-and-forward protocol with one relay node, the relay normalizes the received signal and then amplifies it before re-transmission.

The problem with the previous protocols is the loss in the data rate as the number of relays increases and this leads to the use of what is known as distributed space-time coding (DSTC) [3]. DSTC are designed to achieve diversity over flat, frequency non-selective, fading channels. For the case of multipath fading channels, the design of distributed spacefrequency codes (DSFCs) is crucial to exploit the frequency diversity of the channel. The presence of multipaths provides another mean for achieving diversity across the frequency axis. Exploiting the frequency axis diversity can highly improve the system performance, by achieving higher diversity orders.

The design of DSFCs was considered in [4] in which DSFCs, based on OFDM modulation, were designed for a system employing the DAF protocol. The authors assume that all the relays always decode correctly, which is not always true especially over wireless fading channels. The presence of the cyclic prefix in OFDM modulation can mitigate relays synchronization errors which is also a property of our proposed OFDM based DSFCs.

In this paper, we consider the design of distributed spacefrequency codes (DSFCs) that can exploit the multipath nature of the wireless relay channels. By exploiting the frequency diversity of the channel higher performance gains are achievable especially at high signal-to-noise ratio (SNR). We consider the use of the DAF protocol as the user cooperation protocol. We derive sufficient conditions for the proposed code structure to achieve full diversity. We prove that the proposed DSFC can achieve full diversity of order $N L$ where $N$ is the number of cooperating relay nodes and $L$ is the number of paths per channel.

Notations: $\lfloor x\rfloor$ denotes the largest integer that is less than $x$. $\operatorname{diag}(\mathbf{y})$, where $\mathbf{y}$ is a $T \times 1$ vector, is the $T \times T$ diagonal matrix with the elements of $\mathbf{y}$ on its diagonal. $\mathbf{A} \otimes \mathbf{B}$ denotes the tensor product of the two matrices $\mathbf{A}$ and $\mathbf{B}$. $\|\mathbf{A}\|_{F}^{2}$ of the $m \times n$ matrix $\mathbf{A}$ is the Frobenius norm of the matrix defined as $\|\mathbf{A}\|_{F}^{2}=\sum_{i=1}^{m} \sum_{j=1}^{n}|\mathbf{A}(i, j)|^{2}$.

\section{SySTEM MODEL}

In this section, the system model for the DSFCs with the DAF protocol is presented. A two-hop relay channel model, where there is no direct link from the source node to the destination node, is used. A simplified system model is depicted in Fig. 1. The system is based on OFDM modulation with $K$ subcarriers. The channel between the source node and the $n$-th relay node is modeled as a multipath fading channel 


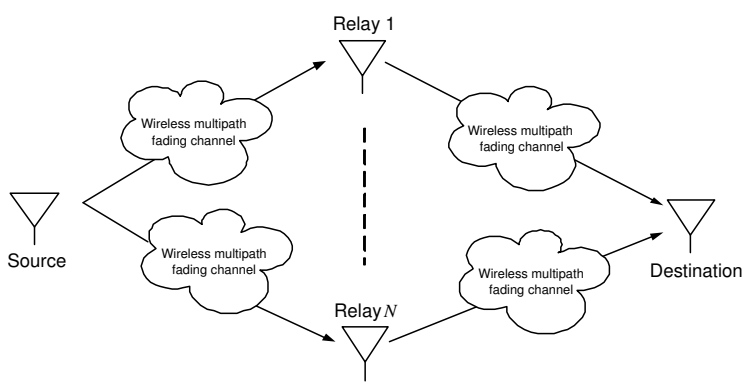

Fig. 1. Simplified system model for the distributed space-frequency codes.

with $L$ paths as

$$
h_{s, r_{n}}(\tau)=\sum_{l=1}^{L} \alpha_{s, r_{n}}(l) \delta\left(\tau-\tau_{l}\right),
$$

where $\tau_{l}$ is the delay of the $l$-th path, $\delta(\cdot)$ is the Dirac delta function, and $\alpha_{s, r_{n}}(l)$ is the complex amplitude of the $l$ th path. The $\alpha_{s, r_{n}}(l)$ 's are modeled as zero-mean complex Gaussian random variables with variance $E\left[\left|\alpha_{s, r_{n}}(l)\right|^{2}\right]=$ $\sigma^{2}(l)$, where we assume symmetry between the relay nodes for simplicity of presentation. The analysis can be easily extended to the asymmetric case. The channels are normalized such that the channel variance $\sum_{l=1}^{L} \sigma^{2}(l)=1$.

The channel frequency response is given by

$$
H_{s, r_{n}}(f)=\sum_{l=1}^{L} \alpha_{s, r_{n}}(l) e^{-j 2 \pi f \tau_{l}} .
$$

A cyclic prefix is introduced to convert the multipath frequency-selective fading channels to flat fading subchannels on the subcarriers.

The system has two phases, as follows. In phase 1 , if $N$ relays are assigned for helping the source node, the source node broadcasts the information to the $N$ relays. The received signal in the frequency domain on the $k$-th subcarrier at the $n$-th relay node is given by

$$
y_{s, r_{n}}(k)=\sqrt{P_{s}} H_{s, r_{n}}(k) s(k)+\eta_{s, r_{n}}(k), \quad k=1, \cdots, K,
$$

where $P_{s}$ is the transmitted source node power, $H_{s, r_{n}}(k)$ is the channel attenuation of the source node to the $n$-th relay node channel on the $k$-th subcarrier, $s(k)$ is the transmitted source node symbol on the $k$-th subcarrier with $E\left\{|s(k)|^{2}\right\}=1$, and $\eta_{s, r_{n}}(k)$ is the $n$-th relay node additive white Gaussian noise on the $k$-th subcarrier. $\eta_{s, r_{n}}(k)$ is modeled as zero-mean circularly symmetric complex Gaussian random variable with variance $N_{0} / 2$ per dimension. The subcarrier noise terms are statistically independent assuming that the time domain noise samples are statistically independent ${ }^{1} . H_{s, r_{n}}(k)$ is given by

$$
H_{s, r_{n}}(k)=\sum_{l=1}^{L} \alpha_{s, r_{n}}(l) e^{-j 2 \pi(k-1) \Delta f \tau_{l}}, k=1, \cdots, K,
$$

${ }^{1} \mathrm{FFT}$, which is used to transform the received data from the time domain to the frequency domain, can be represented by a unitary matrix multiplication. Unitary transformations of a Gaussian random vector, whose components are statistically independent, results in a Gaussian random vector with statistically independent components. where $\Delta f=1 / T$ is the subcarrier frequency separation and $T$ is the OFDM symbol duration. We assume perfect channel state information at any receiving node but no channel information at transmitting nodes.

In phase 2, relays that have decoded correctly in phase 1 will forward the source node information. Each relay is assumed to be able to decide whether it has decoded correctly or not. This can be achieved through the use of either error detecting codes or the use of SNR thresholds at the relay nodes. Relays will only forward the source node information if the received SNR is larger than the threshold [5].

The transmitted $K \times N$ space-frequency (SF) codeword from the relay nodes is given by

$$
\mathbf{C}_{r}=\left(\begin{array}{cccc}
C_{r}(1,1) & C_{r}(1,2) & \cdots & C_{r}(1, N) \\
C_{r}(2,1) & C_{r}(2,2) & \cdots & C_{r}(2, N) \\
\vdots & \vdots & \ddots & \vdots \\
C_{r}(K, 1) & C_{r}(K, 2) & \cdots & C_{r}(K, N)
\end{array}\right)
$$

where $C_{r}(k, n)$ is the symbol transmitted by the $n$-th relay node on the $k$-th subcarrier. The SF is assumed to satisfy the power constraint $\left\|\mathbf{C}_{r}\right\|_{F}^{2} \leq K$.

The received signal at the destination node on the $k$-th subcarrier is given by

$$
y_{d}(k)=\sqrt{P_{r}} \sum_{n=1}^{N} H_{r_{n}, d}(k) C_{r}(k, n) I_{n}+\eta_{r_{n}, d}(k),
$$

where $P_{r}$ is the relay node power, $H_{r_{n}, d}(k)$ is the attenuation of the channel between the $n$-th relay node and the destination node on the $k$-th subcarrier, $\eta_{r_{n}, d}(k)$ is the destination additive white Gaussian noise on the $k$-th subcarrier, and $I_{n}$ is the state of the $n$-th relay. $I_{n}$ will equal 1 if the $n$-th relay has decoded correctly in phase 1 , otherwise, $I_{n}$ will equal 0.

\section{Performance Analysis}

It is now necessary to develop sufficient code design criteria for the DSFC to achieve full diversity of order $N L$. The proposed DSFCs will have two stages of coding: the first stage is coding at the source node and the second stage is coding at the relay nodes.

\section{A. Source Node Coding}

The transmitted source node code will be designed to guarantee a diversity of order $L$ at the relay nodes, and this will in turn cause the proposed DSFC to achieve full diversity of order $N L$ as will be shown later. Due to the symmetry assumption, the pairwise error probability (PEP) is the same at any relay node. For two distinct transmitted source node symbols, $\mathbf{s}$ and $\tilde{\mathbf{s}}$, the PEP can be tightly upper bounded as [6], [7]

$$
P E P(\mathbf{s} \rightarrow \tilde{\mathbf{s}}) \leq\left(\begin{array}{c}
2 \nu-1 \\
\nu
\end{array}\right)\left(\prod_{i=1}^{\nu} \lambda_{i}\right)^{-1}\left(\frac{P_{s}}{N_{0}}\right)^{-\nu}
$$

and $\nu$ is the rank of the matrix $\mathbf{C} \circ \mathbf{R}$ where

$$
\mathbf{C}=(\mathbf{s}-\tilde{\mathbf{s}})(\mathbf{s}-\tilde{\mathbf{s}})^{\mathcal{H}},
$$




$$
\mathbf{R}=E\left\{\mathbf{H}_{s, r_{n}} \mathbf{H}_{s, r_{n}}^{\mathcal{H}}\right\}
$$

and $\mathbf{H}_{s, r_{n}}=\left[H_{s, r_{n}}(1), \cdots, H_{s, r_{n}}(K)\right]^{T} . \lambda_{i}$ 's are the nonzero eigenvalues of the matrix $\mathbf{C} \circ \mathbf{R}$, where $\circ$ denotes the Hadamard product ${ }^{2}$.

The correlation matrix, $\mathbf{R}$, of the channel impulse response can be found as

$$
\begin{aligned}
\mathbf{R} & =E\left\{\mathbf{H}_{s, r_{n}} \mathbf{H}_{s, r_{n}}^{\mathcal{H}}\right\} \\
& =\mathbf{W E}\left\{\alpha_{s, r_{n}} \alpha_{s, r_{n}}^{\mathcal{H}}\right\} \mathbf{W}^{\mathcal{H}} \\
& =\mathbf{W} \operatorname{diag}\left\{\sigma^{2}(1), \sigma^{2}(2), \cdots, \sigma^{2}(L)\right\} \mathbf{W}^{\mathcal{H}},
\end{aligned}
$$

where

$$
\begin{gathered}
\alpha_{s, r_{n}}=\left[\alpha_{s, r_{n}}(1), \alpha_{s, r_{n}}(2), \cdots, \alpha_{s, r_{n}}(L)\right]^{T} \\
\mathbf{W}=\left(\begin{array}{cccc}
1 & 1 & \cdots & 1 \\
w^{\tau_{1}} & w^{\tau_{2}} & \cdots & w^{\tau_{L}} \\
\vdots & \vdots & \ddots & \vdots \\
w^{(K-1) \tau_{1}} & w^{(K-1) \tau_{2}} & \cdots & w^{(K-1) \tau_{L}}
\end{array}\right),
\end{gathered}
$$

and $w=e^{-j 2 \pi \Delta f}$.

The coding at the source node is implemented to guarantee a diversity of order $L$, which is the maximum achievable diversity order at the relay nodes. The transmitted $K \times 1$ source node code is partitioned into subblocks of length $L$. Let $M=\lfloor K / L\rfloor$ denote the number of subblocks in the source node transmitted OFDM block. The transmitted $K \times 1$ source node code is given as

$\mathbf{s}=[s(1), s(2), \cdots, s(K)]^{T}=\left[\mathbf{F}_{1}^{T}, \mathbf{F}_{2}^{T}, \cdots, \mathbf{F}_{M}^{T}, \mathbf{0}_{K-M L}^{T}\right]^{T}$,

where $\mathbf{F}_{i}=\left[F_{i}(1), \cdots, F_{i}(L)\right]^{T}$ is the $i$-th subblock of dimension $L \times 1$. Zeros are padded if $K$ is not an integer multiple of $L$. For any two distinct source codewords, $\mathbf{s}$ and $\tilde{\mathbf{s}}=\left[\tilde{\mathbf{F}}_{1}^{T}, \tilde{\mathbf{F}}_{2}^{T}, \cdots, \tilde{\mathbf{F}}_{M}^{T}, \mathbf{0}_{K-M L}^{T}\right]^{T}$, at least one index $p_{0}$ exists for which $\mathbf{F}_{p_{0}}$ is not equal to $\tilde{\mathbf{F}}_{p_{0}}$.

Based on the proposed structure of the transmitted code from the source node, sufficient conditions for the code to achieve a diversity of order $L$ at the relay nodes are derived. We assume for $\mathbf{s}$ and $\tilde{\mathbf{s}}$ that $\mathbf{F}_{p}=\tilde{\mathbf{F}}_{p}$ for all $p \neq p_{0}$. This does not decrease the rank of the matrix $\mathbf{C} \circ \mathbf{R}$ [7]. Define the $L \times L$ matrix $\mathbf{Q}=\left\{q_{i, j}\right\}$ as $q_{i, j}=\sum_{l=1}^{L} \sigma^{2}(l) w^{(i-j) \tau(l)}, \quad 1 \leq$ $i, j \leq L$. Note that the non-zero eigenvalues of the matrix $\mathbf{C} \circ \mathbf{R}$ are the same as those of the matrix $\left(\mathbf{F}_{p_{0}}-\tilde{\mathbf{F}}_{p_{0}}\right)$

$$
\begin{aligned}
& \left(\mathbf{F}_{p_{0}}-\tilde{\mathbf{F}}_{p_{0}}\right)^{\mathcal{H}} \circ \mathbf{Q} \text {. Hence, we have } \\
& \quad\left(\mathbf{F}_{p_{0}}-\tilde{\mathbf{F}}_{p_{0}}\right)\left(\mathbf{F}_{p_{0}}-\tilde{\mathbf{F}}_{p_{0}}\right)^{\mathcal{H}} \circ \mathbf{Q} \\
& \quad=\left[\operatorname{diag}\left(\mathbf{F}_{p_{0}}-\tilde{\mathbf{F}}_{p_{0}}\right) \mathbf{1}_{L \times L} \operatorname{diag}\left(\mathbf{F}_{p_{0}}-\tilde{\mathbf{F}}_{p_{0}}\right)^{\mathcal{H}}\right] \circ \mathbf{Q} \\
& \quad=\operatorname{diag}\left(\mathbf{F}_{p_{0}}-\tilde{\mathbf{F}}_{p_{0}}\right) \mathbf{Q} \operatorname{diag}\left(\mathbf{F}_{p_{0}}-\tilde{\mathbf{F}}_{p_{0}}\right)^{\mathcal{H}}
\end{aligned}
$$

\footnotetext{
${ }^{2}$ If $\mathbf{A}=\left\{a_{i, j}\right\}$ and $\mathbf{B}=\left\{b_{i, j}\right\}$ are two $m \times n$ matrices, the Hadamard product is defined as $\mathbf{D}=\mathbf{A} \circ \mathbf{B}=\left\{d_{i, j}\right\}$, where $d_{i, j}=a_{i, j} b_{i, j}$.
}

where $\mathbf{1}_{L \times L}$ is the $L \times L$ matrix whose all elements are ones. The last equality follows from a property of the Hadamard product ([8], p.304).

If all of the eignenvalues of the matrix $\left(\mathbf{F}_{p_{0}}-\tilde{\mathbf{F}}_{p_{0}}\right)\left(\mathbf{F}_{p_{0}}-\tilde{\mathbf{F}}_{p_{0}}\right)^{\mathcal{H}} \circ \mathbf{Q}$ are non-zero, then their product can be calculated as

$$
\begin{aligned}
& \operatorname{det}\left(\left(\mathbf{F}_{p_{0}}-\tilde{\mathbf{F}}_{p_{0}}\right)\left(\mathbf{F}_{p_{0}}-\tilde{\mathbf{F}}_{p_{0}}\right)^{\mathcal{H}} \circ \mathbf{Q}\right) \\
& =\operatorname{det}\left(\operatorname{diag}\left(\mathbf{F}_{p_{0}}-\tilde{\mathbf{F}}_{p_{0}}\right)\right) \operatorname{det}(\mathbf{Q}) \operatorname{det}\left(\operatorname{diag}\left(\mathbf{F}_{p_{0}}-\tilde{\mathbf{F}}_{p_{0}}\right)^{\mathbf{H}}\right) \\
& =\prod_{l=1}^{L}\left|F_{p_{0}}(l)-\tilde{F}_{p_{0}}(l)\right|^{2}(\operatorname{det}(Q)) .
\end{aligned}
$$

The matrix $\mathbf{Q}$ is non-singular. Hence, if the product $\prod_{l=1}^{L}\left|F_{p_{0}}(l)-\tilde{F}_{p_{0}}(l)\right|^{2}$ is non-zero over all possible pairs of distinct transmitted source codewords, $\mathbf{s}$ and $\tilde{\mathbf{s}}$, then a diversity of order $L$ will be achieved at each relay node.

In phase 2 , relays that have decoded correctly in phase 1 will forward the source node information. The received signal at the destination node on the $k$-th subcarrier is as given in (6). The state of the $n$-th relay node $I_{n}$ is a Bernoulli random variable with a probability mass function (pmf) given by

$$
I_{n}= \begin{cases}0 & \text { with probability }=S E R \\ 1 & \text { with probability }=1-S E R,\end{cases}
$$

where $S E R$ is the symbol error rate at the $n$-th relay node. Note that $S E R$ is the same for any relay node due to the symmetry assumption. If the transmitted code from the source node is designed such that the product $\prod_{l=1}^{L}\left|F_{p_{0}}(l)-\tilde{F}_{p_{0}}(l)\right|^{2}$ is non-zero, for at least one index $p_{0}$, over all the possible pairs of distinct transmitted source codewords, $\mathbf{s}$ and $\tilde{\mathbf{s}}$, then the $S E R$ at the $n$-th relay node can be upper bounded as

$$
\begin{aligned}
S E R & =\sum_{\mathbf{s} \in \mathcal{S}} \operatorname{Pr}\{\mathbf{s}\} \operatorname{Pr}\{\text { error given that } \mathbf{s} \text { was transmitted }\} \\
& \leq \sum_{\mathbf{s} \in \mathcal{S}} \operatorname{Pr}\{\mathbf{s}\} \sum_{\tilde{\mathbf{s}} \in \mathcal{S}, \tilde{\mathbf{s}} \neq \mathbf{s}} \operatorname{PEP}(\mathbf{s} \rightarrow \tilde{\mathbf{s}}) \\
& \leq c \times S N R^{-L},
\end{aligned}
$$

where $\mathcal{S}$ is the set of all possible transmitted source codewords and $c$ is a constant that does not depend on the $S N R$. The first inequality follows from the union upper bound and the second inequality follows from (7), where $S N R$ is defined as $S N R=P_{s} / N_{0}$.

\section{B. Relay Nodes Coding}

Next, the design of the SF code at the relay nodes to achieve a diversity of order $N L$ is considered. We propose to design SF codes constructed from the concatenation of block diagonal matrices, which is similar to the structure used in [7] to design full-rate, full-diversity space-frequency codes. The 
only difference between our system and the SF coding done in [7] comes from the fact that in our system not all of the relay nodes will always transmit their parts of the SF code, while in [7], all of the antennas will always transmit their codes.

Let $P=\lfloor K / N L\rfloor$ denote the number of subblocks in the transmitted OFDM block from the relay nodes. The transmitted $K \times N$ SF codeword from the relay nodes, if all relays decoded correctly, is given by

$$
\mathbf{C}_{r}=\left[\mathbf{G}_{1}^{T}, \mathbf{G}_{2}^{T}, \cdots, \mathbf{G}_{P}^{T}, \mathbf{0}_{K-P L N}^{T}\right]^{T},
$$

where $\mathbf{G}_{i}$ is the $i$-th subblock of dimension $N L \times N$. Zeros are padded if $K$ is not an integer multiple of $N L$. Each $\mathbf{G}_{i}$ is a block diagonal matrix that has the structure

$$
\mathbf{G}_{i}=\left(\begin{array}{cccc}
\mathbf{X}_{1_{L \times 1}} & \mathbf{0}_{L \times 1} & \cdots & \mathbf{0}_{L \times 1} \\
\mathbf{0}_{L \times 1} & \mathbf{X}_{2_{L \times 1}} & \cdots & \mathbf{0}_{L \times 1} \\
\vdots & \vdots & \ddots & \vdots \\
\mathbf{0}_{L \times 1} & \mathbf{0}_{L \times 1} & \cdots & \mathbf{X}_{N_{L \times 1}}
\end{array}\right)
$$

and let $\mathbf{X}=\left[\mathbf{X}_{1}^{T}, \mathbf{X}_{2}^{T}, \cdots, \mathbf{X}_{N}^{T}\right]=[x(1), x(2), \cdots, x(N L)]$.

For two distinct transmitted source codwords, $\mathbf{s}$ and $\tilde{\mathbf{s}}$, and a given realization of the relays states $\mathbf{I}=\left[I_{1}, I_{2}, \cdots, I_{n}\right]^{T}$, the conditional PEP can be tightly upper bounded as

$$
\operatorname{PEP}(\mathbf{s} \rightarrow \tilde{\mathbf{s}} / \mathbf{I}) \leq\left(\begin{array}{c}
2 \kappa-1 \\
\kappa
\end{array}\right)\left(\prod_{i=1}^{\kappa} \eta_{i}\right)^{-1}\left(\frac{P_{r}}{N_{0}}\right)^{-\kappa}
$$

and $\kappa$ is the rank of the matrix $\mathbf{C}(\mathbf{I}) \circ \mathbf{R}$ where

$$
\mathbf{C}(\mathbf{I})=\left(\mathbf{C}_{r}-\tilde{\mathbf{C}}_{r}\right) \operatorname{diag}(\mathbf{I})\left(\mathbf{C}-\tilde{\mathbf{C}}_{r}\right)^{\mathcal{H}} .
$$

For two source codewords, $\mathbf{s}$ and $\tilde{\mathbf{s}}$, at least one index $p_{0}$ exists for which $\mathbf{G}_{p_{0}} \neq \tilde{\mathbf{G}}_{p_{0}}$. We assume for $\mathbf{s}$ and $\tilde{\mathbf{s}}$ that $\mathbf{G}_{p}=\tilde{\mathbf{G}}_{p}$ for all $p \neq p_{0}$. As for the source node coding case, this does not decrease the rank of the matrix $\mathbf{C}(\mathbf{I}) \circ \mathbf{R}$ that corresponds to any realization $\mathbf{I}$ of the relays states.

Define the $N L \times N L$ matrix $\mathbf{S}=\left\{s_{i, j}\right\}$ as

$$
s_{i, j}=\sum_{l=1}^{L} \sigma^{2}(l) w^{(i-j) \tau(l)}, \quad 1 \leq i, j \leq N L .
$$

Note that the non-zero eigenvalues of the matrix $\mathbf{C}(\mathbf{I}) \circ \mathbf{R}$ are the same as the non-zero eigenvalues of the matrix $\left(\mathbf{G}_{p_{0}}(\mathbf{I})-\tilde{\mathbf{G}}_{p_{0}}(\mathbf{I})\right)\left(\mathbf{G}_{p_{0}}(\mathbf{I})-\tilde{\mathbf{G}}_{p_{0}}(\mathbf{I})\right)^{\mathcal{H}} \circ \mathbf{S}$ where $\mathbf{G}_{p_{0}}(\mathbf{I})$ is formed from $\mathbf{G}_{p_{0}}$ by setting the columns corresponding to the relays that have decoded erroneously to zeros. Hence,

$$
\begin{aligned}
& \left(\mathbf{G}_{p_{0}}(\mathbf{I})-\tilde{\mathbf{G}}_{p_{0}}(\mathbf{I})\right)\left(\mathbf{G}_{p_{0}}(\mathbf{I})-\tilde{\mathbf{G}}_{p_{0}}(\mathbf{I})\right)^{\mathcal{H}} \circ \mathbf{S} \\
& =\left(\operatorname{diag}(\mathbf{X}-\tilde{\mathbf{X}})\left(\operatorname{diag}(\mathbf{I}) \otimes \mathbf{1}_{L \times 1}\right)\left(\operatorname{diag}(\mathbf{I}) \otimes \mathbf{1}_{L \times 1}\right)^{\mathcal{H}}\right. \\
& \left.\times \operatorname{diag}(\mathbf{X}-\tilde{\mathbf{X}})^{\mathcal{H}}\right) \circ \mathbf{S} \\
& =\left(\operatorname{diag}(\mathbf{X}-\tilde{\mathbf{X}})\left(\operatorname{diag}(\mathbf{I}) \otimes \mathbf{1}_{L \times L}\right) \operatorname{diag}(\mathbf{X}-\tilde{\mathbf{X}})^{\mathcal{H}}\right) \circ \mathbf{S} \\
& =\operatorname{diag}(\mathbf{X}-\tilde{\mathbf{X}})\left[\left(\operatorname{diag}(\mathbf{I}) \otimes \mathbf{1}_{L \times L}\right) \circ \mathbf{S}\right] \operatorname{diag}(\mathbf{X}-\tilde{\mathbf{X}})^{\mathcal{H}},
\end{aligned}
$$

where the second and the third equalities follow from the properties of the tensor and Hadamard products [7], [8].

Let $n_{\mathbf{I}}=\sum_{n=1}^{N} I_{n}$ denote the number of relays that have decoded correctly corresponding to a realization $\mathbf{I}$ of the relays states. Using (17), the product of the non-zero eigenvalues of the matrix $\mathbf{C}(\mathbf{I}) \circ \mathbf{R}$ can be found as

$$
\prod_{i=1}^{\kappa} \eta_{i}=\left(\prod_{i=1, i \in \mathcal{I}}^{N L}|x(i)-\tilde{x}(i)|^{2}\right) \cdot\left(\operatorname{det}\left(\mathbf{S}_{0}\right)\right)^{n_{\mathbf{I}}}
$$

where $\mathcal{I}$ is the index set of symbols that are transmitted from the relays that have decoded correctly corresponding to the realization $\mathbf{I}$ and $\mathbf{S}_{0}=\left\{s_{i, j}\right\}, 1 \leq i, j \leq L$. The result in (18) is based on the assumption that the product $\prod_{i=1, i \in \mathcal{I}\}}^{N L} \mid x(i)-$ $\left.\tilde{x}(i)\right|^{2}$ is non-zero. The first product in (18) is over $n_{\mathbf{I}} L$ terms. The matrix $\mathbf{S}_{0}$ is always full rank of order $L$. Hence, designing the product $\prod_{i=1, i \in \mathcal{I}\}}^{N L}|x(i)-\tilde{x}(i)|^{2}$ to be non-zero will guarantee a rate of decay, at high SNR, of the conditional PEP as $S N R^{-n_{\mathbf{I}} L}$, where SNR is now defined as $S N R=P_{r} / N_{0}$. To guarantee that this rate of decay, $S N R^{-n_{\mathbf{I}} L}$, is always achieved irrespective of the state realization $\mathbf{I}$ of the relay nodes then the product $\prod_{i=1}^{N L}|x(i)-\tilde{x}(i)|^{2}$ should be non-zero. Hence, designing the product $\prod_{i=1}^{N L}|x(i)-\tilde{x}(i)|^{2}$ to be nonzero for any pair of distinct source codewords is a sufficient condition for the conditional PEP to decay as $S N R^{-n_{\mathbf{I}} L}$ for any realization $\mathbf{I}$, where $n_{\mathbf{I}}$ is the number of relays that have decoded correctly corresponding to $\mathbf{I}$.

Now, we calculate the PEP at the destination node for our proposed DSFC structure. Let $c_{r}$ denote the number of relays that have decoded correctly. Then $c_{r}$ follows a Binomial distribution as ${ }^{3}$

$$
\operatorname{Pr}\left\{c_{r}=k\right\}=\left(\begin{array}{c}
N \\
k
\end{array}\right)(1-S E R)^{k} S E R^{N-k},
$$

where $S E R$ is the symbol error rate at the relay nodes. The destination PEP is given by

$$
\begin{aligned}
& \operatorname{PEP}(\mathbf{s} \rightarrow \tilde{\mathbf{s}}) \\
& =\sum_{\mathbf{I}} \operatorname{Pr}\{\mathbf{I}\} \operatorname{PEP}(\mathbf{s} \rightarrow \tilde{\mathbf{s}} / \mathbf{I}) \\
& =\sum_{k=0}^{N} \operatorname{Pr}\left\{c_{r}=k\right\} \sum_{\left\{\mathbf{I}: n_{\mathbf{I}}=k\right\}} \operatorname{PEP}(\mathbf{s} \rightarrow \tilde{\mathbf{s}} / \mathbf{I}) \\
& =\sum_{k=0}^{N}\left(\begin{array}{c}
N \\
k
\end{array}\right)(1-S E R)^{k} S E R^{N-k} \sum_{\left\{\mathbf{I}: n_{\mathbf{I}}=k\right\}} P E P(\mathbf{s} \rightarrow \tilde{\mathbf{s}} / \mathbf{I}),
\end{aligned}
$$

Using the upper bound on the $S E R$ at the relay nodes given in (13) and the expression for the conditional PEP at the destination node in (16), and upper bounding $(1-S E R)$ by 1 , we can easily show that

$$
P E P(\mathbf{s} \rightarrow \tilde{\mathbf{s}}) \leq \text { constant } \times S N R^{-N L} .
$$

${ }^{3} c_{r}$ is a Binomial random variable as it is the sum of independent identically distributed Bernoulli random variables. 
Hence, our proposed structure for DSFCs with two-stage coding at the source node and the relay nodes achieves a diversity of order $N L$, which is the rate of decay of the PEP at high SNR.

For coding at the source and relay nodes we will use a linear mapping to form the transmitted subblocks, $\mathbf{D}=\mathbf{V}_{T \times T} \mathbf{s}_{\mathbf{G}}$, where $\mathbf{s}_{\mathbf{G}}$ is a $T \times 1$ source symbols vector. $\mathbf{s}_{\mathbf{G}}$ is carved from QAM or PSK constellations. The goal is to maximize the product $\min _{\mathbf{s} \neq \tilde{\mathbf{s}}} \prod_{m=1}^{T}|\mathbf{D}(m)-\tilde{\mathbf{D}}(m)|^{2}$, where s and $\tilde{\mathbf{s}}$ are two distinct source symbols vectors. It was proposed in [9] and [10] to use both Hadamard transforms and Vandermonde matrices to design the $\mathbf{V}_{T \times T}$ matrix. The transforms based on the Vandermonde matrices proved to give larger minimum product distance than the Hadamard based transforms. Two classes of optimum transforms were proposed in [9] as follows.

1) If $T=2^{k}(k \geq 1)$, the optimum transform is given by $\mathbf{V}_{\text {opt }}=\frac{1}{\sqrt{T}} \operatorname{vander}\left(\theta_{1}, \theta_{2}, \ldots, \theta_{T}\right)$, where $\theta_{1}, \theta_{2}, \ldots, \theta_{T}$ are $\theta_{n}=e^{j \frac{4 n-3}{2 T} \pi}, \quad n=1,2, \ldots, T$.

2) If $T=3.2^{k}(k \geq 0)$, the optimum transform is given by $\mathbf{V}_{\text {opt }}=\frac{1}{\sqrt{T}} \operatorname{vander}\left(\theta_{1}, \theta_{2}, \ldots, \theta_{T}\right)$, where $\theta_{1}, \theta_{2}, \ldots, \theta_{T}$ are $\theta_{n}=e^{j \frac{6 n-1}{3 T} \pi}, \quad n=1,2, \ldots, T$.

\section{Simulation Results}

In this section, we present some simulation results for the proposed DSFCs. In all simulations, the source is assumed to have two relay nodes helping in forwarding its information. Fig. 2 shows the case of a simple two-ray $L=2$ with a delay $\tau=5 \mu \mathrm{sec}$ between the two rays and the case of $L=4$ with a delay profile given as $[0,5 \mu \mathrm{sec}, 10 \mu \mathrm{sec}, 15 \mu \mathrm{sec}]$. For the case of $L=2$, the two rays have equal variances, i.e., $\sigma^{2}(1)=\sigma^{2}(2)$. For the case of $L=4$, the rays are modeled to have equal powers, i.e., $\sigma^{2}(l)=\sigma^{2}, l=1, \cdots, 4$. The number of subcarriers is $K=128$ with a system bandwidth of $1 \mathrm{MHz}$. We use BPSK modulation and Vandermonde based linear transformations. Fig. 2 shows the SER of the proposed DSFCs versus the SNR defined as $S N R=\frac{P_{s}+P_{r}}{N_{0}}$, and we use $P_{s}=P_{r}$. We simulated three cases: all channel variances are ones, relays close to source, and relays close to destination. For the case of relays close to source, the variance of any source-relay channel is taken to be 10 and the variance of any relay-destination channel is taken to be 1 . For the case of relays close to destination, the variance of any source-relay channel is taken to be 1 and the variance of any relay-destination channel is taken to be 10. For the case of $L=2$, the proposed DSFC achieves a diversity of order $N L=4$ and for the case of $L=4$, it achieves a diversity of order $N L=8$.

\section{CONCLUSION}

In this paper, the design of distributed space-frequency codes (DSFCs) was considered for the wireless multipath relay channels. The use of DSFCs can highly improve the system performance by achieving higher order diversities by exploiting the multipath diversity of the channel as well as the cooperative diversity. For DSFCs with the DAF protocol, we have proposed a two stages coding scheme: source node

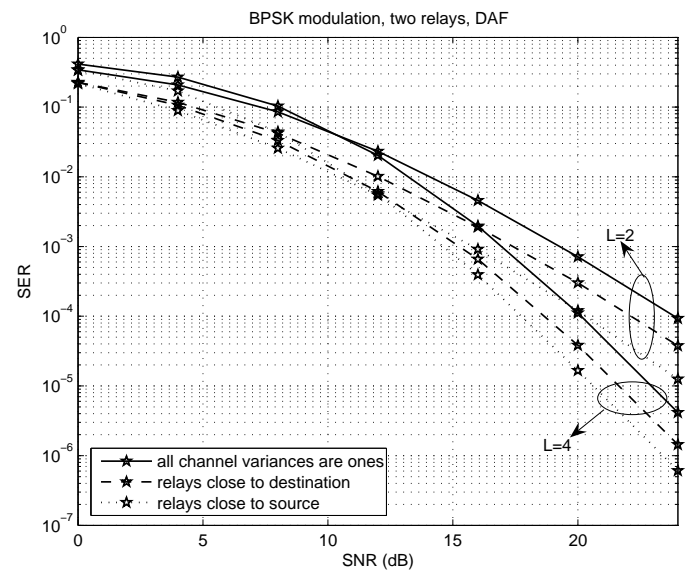

Fig. 2. SER for DSFCs, for BPSK modulation with Vandermonde based linear transformations, versus SNR.

coding and relay nodes coding. We have derived sufficient conditions for the proposed code structure to achieve full diversity of order $N L$ where $N$ is the number of relay nodes and $L$ is the number of multipaths per channel.

The proposed DSFCs are robust against the synchronization errors caused by the relays' timings mismatches and propagation delays due to the presence of the cyclic prefix in the OFDM transmission. Also, the proposed DSFCs are robust against the relays' carrier offset errors since only one relay is transmitting on any subcarrier at any given instance. These properties of the proposed DSFCs greatly simplifies the system design since it is very difficult to synchronize randomly located relay nodes.

\section{REFERENCES}

[1] A. Sendonaris, E. Erkip, and B. Aazhang, "User cooperation diversityparts I and II," IEEE Trans. Comm., vol. 51, no. 11, pp. 1927-1948, Nov. 2003.

[2] J. N. Laneman, D. N. C. Tse, and G. W. Wornell, "Cooperative diversity in wireless networks: Efficient protocols and outage behavior," IEEE Trans. Info. Theory, vol. 50, no. 12, pp. 3062-3080, December 2004.

[3] J. N. Laneman and G. W. Wornell, "Distributed space-time coded protocols for exploiting cooperative diversity in wireless networks," IEEE Trans. Info. Theory, vol. 49, no. 10, pp. 2415-2425, Oct. 2003.

[4] Y. Li, W. Zhang, and X.-G. Xia, "Distributive high-rate full-diversity space-frequency codes achieving full cooperative and multipath diversity for asynchronous cooperative communications," IEEE Global Communications Conference (GLOBECOM 2006), Dec. 2006.

[5] A. K. Sadek, W. Su, and K. J. R. Liu, "Multi-node cooperative communications in wireless networks," IEEE Trans. Signal Processing, vol. 55, pp. 341-355, Jan 2007.

[6] S. Siwamogsatham, M. P. Fitz, and J. H. Grimm, "A new view of performance analysis of transmit diversity schemes in correlated rayleigh fading," IEEE Trans. Info. Theory, vol. 48, no. 4, pp. 950-956, April 2002.

[7] W. Su, Z. Safar, and K. J. R. Liu, "Full-rate full-diversity spacefrequency codes with optimum coding advantage," IEEE Trans. Info. Theory, vol. 51, no. 1, pp. 229-249, Jan. 2005.

[8] R. A. Horn and C. R. Johnson, Topics in Matrix Analysis, Cambridge Univ. Press, 1991

[9] X. Giraud, E. Boutillon, and J. C. Belfiore, "Algebraic tools to build modulation schemes for fading channels," IEEE Trans. Info. Theory, vol. 43, no. 3, pp. 938-952, May 1997.

[10] J. Boutros and E. Viterbo, "Signal space diversity: A power- and bandwidth-efficient diversity technique for the rayleigh fading channel," IEEE Trans. Info. Theory, vol. 44, no. 4, pp. 1453-1467, July 1998. 